\title{
Neddylation inhibition activates the protective autophagy through NF-KB- catalase-ATF3 Axis in human esophageal cancer cells
}

Yupei Liang ${ }^{1 \dagger}$, Yanyu Jiang ${ }^{1+}$, Xing Jin ${ }^{1 \dagger}$, Ping Chen ${ }^{2}$, Yongqing Heng ${ }^{1}$, Lili Cai ${ }^{1}$, Wenjuan Zhang ${ }^{3}$, Lihui Li $i^{1}$ and Lijun $\mathrm{Jia}^{1 *}$ (D)

\begin{abstract}
Background: Protein neddylation plays a tumor-promoting role in esophageal cancer. Our previous study demonstrated that neddylation inhibition induced the accumulation of ATF4 to promote apoptosis in esophageal cancer cells. However, it is completely unknown whether neddylation inhibition could induce autophagy in esophageal cancer cells and affect the expression of other members of ATF/CREB subfamily, such as ATF3.

Methods: The expression of relevant proteins of NF-KB/Catalase/ATF3 pathway after neddylation inhibition was determined by immunoblotting analysis and downregulated by siRNA silencing for mechanistic studies. ROS generation upon MLN4924 treatment was determined by H2-DCFDA staining. The proliferation inhibition induced by MLN4924 was evaluated by ATPLite assay and apoptosis was evaluated by Annexin V/PI double staining.

Results: For the first time, we reported that MLN4924, a specific inhibitor of Nedd8-activating enzyme, promoted the expression of ATF3 to induce autophagy in esophageal cancer. Mechanistically, MLN4924 inhibited the activity of CRLs and induced the accumulation of its substrate IKBa to block NF-KB activation and Catalase expression. As a result, MLN4924 activated ATF3-induced protective autophagy, thereby inhibiting MLN4924-induced apoptosis, which could be alleviated by ATF3 silencing.
\end{abstract}

Conclusions: In our study, we elucidates a novel mechanism of NF-KB/Catalase/ATF3 pathway in MLN4924-induced protective autophagy in esophageal cancer cells, which provides a sound rationale and molecular basis for combinational anti-ESCC therapy with knockdown ATF3 and neddylation inhibitor (e.g. MLN4924).

Keywords: Neddylation, MLN4924, NF-KB/catalase/ATF3, Autophagy, Apoptosis, Esophageal Cancer

\footnotetext{
* Correspondence: ljiji@shutcm.edu.cn

${ }^{\dagger}$ Yupei Liang, Yanyu Jiang and Xing Jin contributed equally to this work.

${ }^{1}$ Cancer Institute, Longhua Hospital, Shanghai University of Traditional Chinese Medicine, Shanghai 200032, China

Full list of author information is available at the end of the article
}

C C The Author(s). 2020 Open Access This article is licensed under a Creative Commons Attribution 4.0 International License, which permits use, sharing, adaptation, distribution and reproduction in any medium or format, as long as you give appropriate credit to the original author(s) and the source, provide a link to the Creative Commons licence, and indicate if changes were made. The images or other third party material in this article are included in the article's Creative Commons licence, unless indicated otherwise in a credit line to the material. If material is not included in the article's Creative Commons licence and your intended use is not permitted by statutory regulation or exceeds the permitted use, you will need to obtain permission directly from the copyright holder. To view a copy of this licence, visit http://creativecommons.org/licenses/by/4.0/. The Creative Commons Public Domain Dedication waiver (http://creativecommons.org/publicdomain/zero/1.0/) applies to the data made available in this article, unless otherwise stated in a credit line to the data. 


\section{Background}

Post-translational modification of proteins plays crucial roles in the regulation of tumorigenesis and progression. Protein neddylation is an important post-translational modification that adds the ubiquitin-like molecule NEDD8 to substrate proteins [1]. In the process of neddylation, NEDD8 is first catalyzed by Nedd8-activating enzyme (NAE, NAE1 and UBA3 isodimer), transferred to NEDD8 conjugating enzyme E2, and then conjugated to substrates via a specific NEDD8-E3 ligase [2, 3]. The best-characterized substrates of neddylation pathway are the cullin subunits of Cullin-RING E3 ubiquitin ligase (CRL) [2]. As the largest family of E3 ubiquitin ligases, the activation of CRL requires the attachment of NEDD8 to cullin, then to promote ubiquitination and degradation of CRL substrates [4, 5]. Accumulated studies show that protein neddylation is elevated in multiple human cancers and inhibition of this pathway has been developed as a promising anticancer strategy [4, 6-10].

MLN4924, a specific inhibitor of NAE, was previously indentified through a high-throughput screening $[5,11$, 12]. MLN4924 could block cullin neddylation to inhibit the activation of CRLs, thus inducing the accumulation of tumor-suppressive CRL substrates to inhibit tumor growth and metastasis both in vitro and in vivo $[2,4,5$, 13-19]. For its significant anticancer efficacy and welltolerated toxicity, MLN4924 has been advanced into several phase II/III clinical trials against several solid tumors and hematologic malignancies [5, 9, 11, 20, 21]. Mechanistic studies showed that MLN4924 effectively induced DNA re-replication stress/DNA damage response, cellcycle arrest, apoptosis or senescence in a cell-typedependent manner [22-25]. Moreover, MLN4924 also induced pro-survival autophagic responses in cancer cells partially via modulating the HIF1-REDD1-TSC1 or Deptor-mTORC1 pathways [26-28].

Activating transcription factor 3 (ATF3) is a stressresponsive factor that belongs to the ATF/CREB subfamily of the basic region leucine zipper (bZIP) family [29]. ATF3 expression is often correlated with cellular damage and strongly induced by different stress signals. Functionally, ATF3 acts as pro-apoptotic or antiapoptotic signals upon drug treatments in cell contextdependent manners [30-32]. Targeting neddylation has emerged as an attractive anticancer strategy, however, whether and how ATF3 response to neddylationtargeted therapy remains elusive. Here, for the first time, we reported that neddylation inhibition with MLN4924 induces the accumulation of ATF3 to trigger prosurvival autophagy by modulating NF- $\kappa \mathrm{B}-$ Catalase-ROSATF3 axis in esophageal cancer cells, highlighting targeting ATF3-mediated autophagy as a potential strategy to enhance neddylation-targeted anti-ESCC therapy.

\section{Methods}

\section{Cell lines, culture and reagents}

Human ESCC cell lines EC1 and Kyse450 were cultured in Dulbecco's Modifed Eagle's Medium (Hyclone), containing 10\% FBS (Biochrom AG) and 1\% penicillinstreptomycin solution, at $37^{\circ} \mathrm{C}$ with $5 \% \quad \mathrm{CO}_{2}$. Chloroquine (CQ), Bafilomycin A1 (BafA1) and 3methyladenine (3MA) were purchased from Sigma. MLN4924 was synthesized and prepared as previously described.

\section{Cell viability}

Cells were inoculated into 96 well plates $\left(2 \times 10^{3}\right.$ cells per well) and treated with DMSO or MLN4924. According to the manufacturer's protocol, cell proliferation was measured by ATPLite luminescence analysis kit (PerkinElmer).

\section{Immunoblotting}

Cell lysates were prepared for immunoblotting (IB) analysis with antibodies against ATF3 (Cell Signaling Technology), LC3 (Cell Signaling Technology), cleaved PARP (Cell Signaling Technology), ІкB $\alpha$ (Cell Signaling

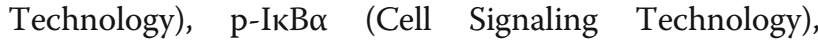
Catalase (Cell Signaling Technology), GAPDH (Cwbiotech) and ACTIN (Cwbiotech). Image J software was used for densitometric analysis.

\section{Gene silencing using siRNA}

EC1 and Kyse450 cells were transfected with siRNA oligonucleotides, synthesized by Ribobio, using Lipofectamine 2000. The sequences of siRNA are as follows:

\section{siATF3: ATGTCCTCTGCGCTGGAAT;}

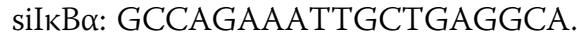

\section{Construction of plasmid}

The pcDNA3-ATF3 plasmid is kindly provided by Prof. Dakang Xu (Ruijin Hospital, Shanghai Jiaotong University School of Medicine, Shanghai) [33]. Briefly, fulllength complementary DNA (cDNA) of ATF3 was cloned into the pcDNA3 vector using standard protocols [33]. Full-length complementary DNA (cDNA) of Catalase was cloned into the $\mathrm{p}-\mathrm{CMV}$ vector using standard protocols. All constructions were confirmed by DNA sequencing before further applications.

RNA isolation and quantitative polymerase chain reaction (Q-PCR).

Total RNA was extracted by Ultrapure RNA kit (Cwbiotech). RNA $(1.0 \mu \mathrm{g})$ was purified and reversely transcribed by PrimeScript ${ }^{\circ}$ RT Master (Takara) according to the manufacturer's instructions. The cDNA was quantifed by real-time quantitative PCR using $\mathrm{SYBR}^{\circ}$ Green Real-Time PCR Master Mixes (Applied 
Biosystems) and a 7500 Real-time PCR system (Applied Biosystems) according to the manufacturer's instructions.

\section{Detection of apoptosis}

Cells were treated with MLN4924 at a specified concentration for appointed time. Apoptosis was detected by Annexin V-FITC/PI apoptosis kit (Biovision, Inc).

\section{Quantifcation of reactive oxygen species}

The quantification of reactive oxygen species(ROS) production was monitored by cell permeable ROS indicator $2^{\prime}, 7^{\prime}$ - dichlorodihydrofuorescein diacetate (H2-DCFDA) (Sigma). The role of ROS in autophagy was evaluated by free radical scavenger NAC (Beyotime). Cells were preincubated with $50 \mu \mathrm{M} \mathrm{NAC}$ for $2 \mathrm{~h}$, then co-incubated with the indicated chemicals and assessed for autophagy or ROS production as described above.

\section{Statistical analysis}

GraphPad prism5 software was used to evaluate the statistical significance of the differences among groups. Unmatched 2-tailed t-test was used to compare the parameters between groups. The level of significance was set at $P<0.05$.

\section{Results}

\section{MLN4924 induced ATF3 transactivation}

Since MLN4924 treatment induced the accumulation of ATF4 [1], a member of ATF/CREB subfamily, in esophageal cancer cells, we reasoned whether neddylation inhibition could affect the expression of other members of ATF/CREB subfamily, such as ATF3. Therefore, we determined the effects of MLN4924 on the expression of ATF3 in EC1 and Kyse450 cells. We found that MLN4924 significantly induced the accumulation of ATF3 in both EC1 and Kyse450 cells (Fig. 1a and Fig. 1b). Given that ATF3 could be ubiquitinated by CRL/ SCF ubiquitin ligase and degraded by proteasome [34], we further determined the stability of ATF3 using cycloheximide (CHX) chase assay upon MLN4924 treatment. Unexpectedly, the half-life of ATF3 was not influenced upon the inactivation of neddylation-CRL axis by MLN4924 (Fig. 1c-d). The expression level of ATF3 was further quantified by densitometric analysis using Image J software. GAPDH was used as the loading control (Fig. 1e-f). Thus, we further determined the expression of ATF3 using real-time PCR for mRNA quantification. As shown in Fig. 1g-h, the expression of ATF3 in transcriptional level was statistically elevated in EC1 and Kyse450 cells upon MLN4924 treatment. These findings collectively demonstrated that MLN4924 induced the transactivation of ATF3.

\section{ATF3 silencing blocked MLN4924-induced autophagy}

We investigated whether and how ATF3 mediates MLN4924-induced cellular responses. As shown, MLN4924 induced the conversion of LC3-I to LC3-II, a classical marker of autophagy, in dose-dependent and time-dependent manner in EC1 and Kyse450 cells (Fig. 2a-b). In addition, we performed autophagic flux analysis by treating cells with classical autophagy inhibitors, including Chloroquine (CQ), bafilomycin A1 (BafA1) and 3-methyladenine (3MA), respectively. As expected, CQ and BafA1 enhanced, while 3MA inhibited the accumulation of LC3-II, indicating that autophagic flux was intact and supraphysiological autophagic response was induced by MLN4924 treatment (Fig. 2c and d). To determine the potential role of ATF3 in MLN4924-induced autophagy, the expression of ATF3 is down-regulated via siRNA silencing. As shown in Fig. 2e-f, the ATF3 knockdown completely blocked the conversion of LC3-I to LC3-II upon MLN4924 treatment, indicating a crucial role of ATF3 in MLN4924induced autophagy in esophageal cancer cells. To further validate the role of ATF3 in increasing autophagy, ATF3 was ectopically expressed in EC1 and Kyse450 cells. Similar to ATF3 accumulation induced by MLN4924 treatment, ATF3 overexpression via transfecting the plasmid of pcDNA3-ATF3 also significantly induced the conversion of LC3-I to LC3-II in a dose-dependent manner in EC1 and Kyse450 cells (Fig. 2g). Taken together, these findings highlight a pivotal role of ATF3 expression in the regulation of autophagy.

\section{MLN4924-induced autophagy was a survival signal and ATF3 silencing enhanced drug toxicity via inducing apoptosis}

Next we determined the role of ATF3-mediated autophagy response upon MLN4924 treatment in esophageal cancer cells. Firstly, we blocked autophagy via both pharmacological (siRNA silencing of autophagy essential gene Beclin1) and genetic approaches (using autophagy inhibitor $\mathrm{CQ}$ ), and found that blocking autophagy significantly enhanced the MLN4924-induced proliferation inhibition in EC1 and Kyse450 cells (Fig. 3a-b), indicating the pro-survival role of MLN4924-induced autophagy. Consistently, the inhibition of autophagic response by siBeclin1 and CQ significantly enhanced MLN4924-induced apoptosis (Fig. 3c-d), as best evidenced by the increase of Annexin V-positive cell populations and the accumulation of cleaved PARP, a classical marker of apoptosis (Fig. 3e). Furthermore, we found that ATF3 knockdown significantly enhanced MLN4924-induced proliferation inhibition (Fig. 3f). In addition, ATF3 knockdown significantly enhanced MLN4924-induced apoptosis, as evidenced by the accumulation of cleaved PARP (Fig. 3g) and 


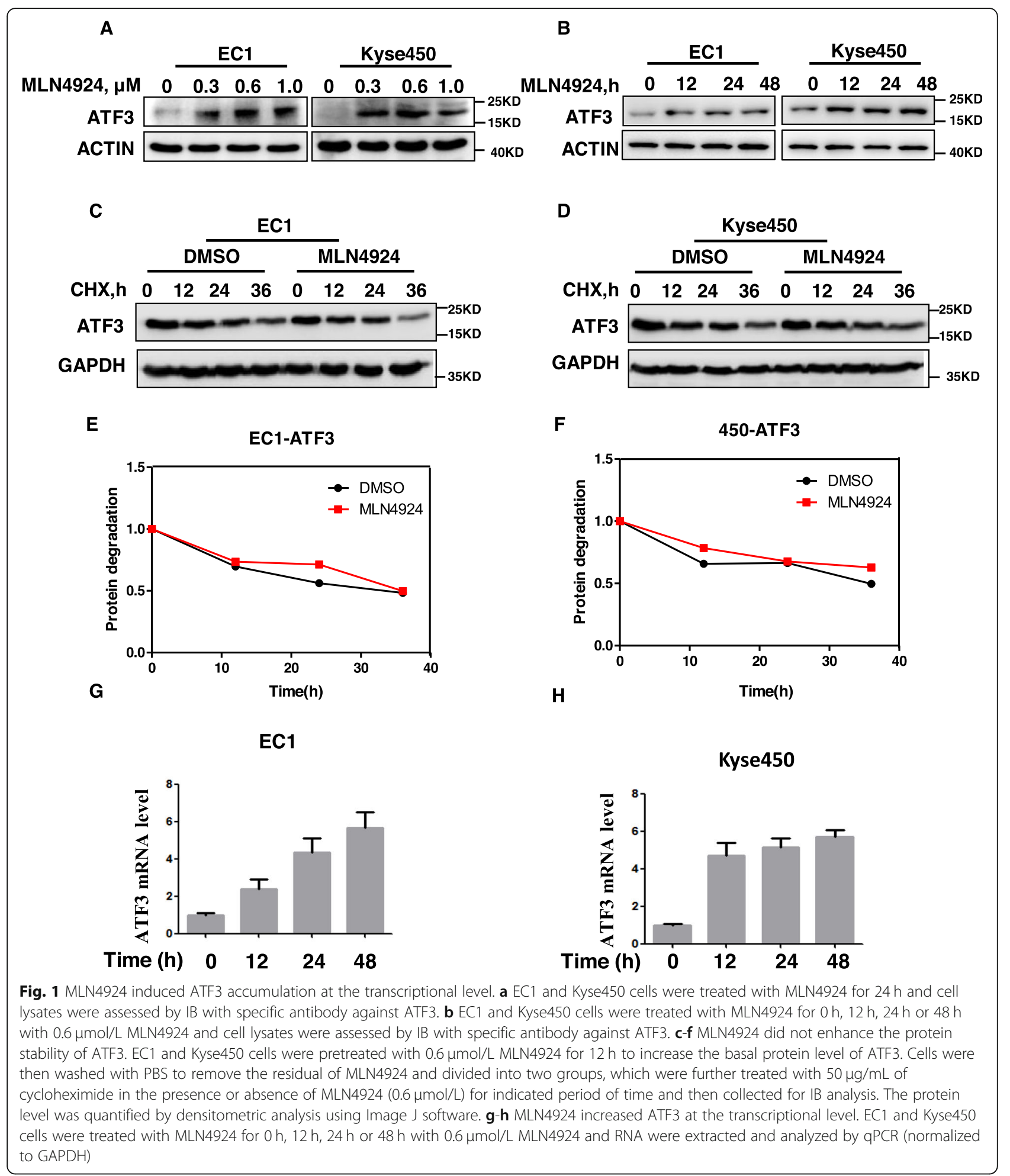

the increase of Annexin V-positive cell populations (Fig. 3h). These results demonstrated that MLN4924 induced the ATF3-mediated autophagy as a prosurvival signal in esophageal cancer cells.
ATF3 accumulation is mediated by ROS upon MLN4924 treatment

Previous studies indicated that ROS could induce ATF3 expression and MLN4924 could induce ROS production 

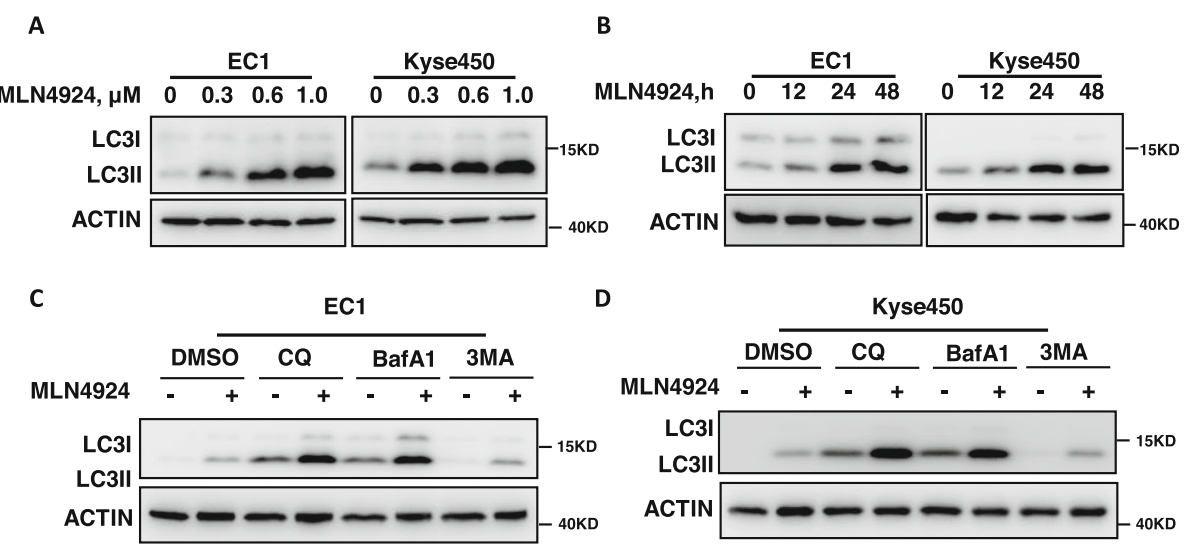

$\mathbf{E}$

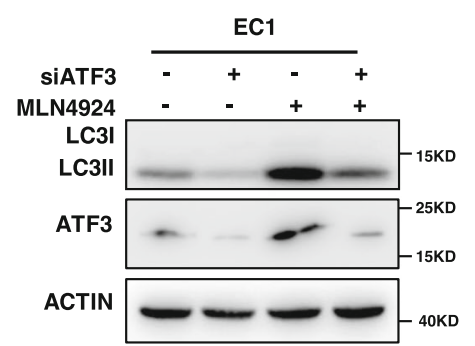

G

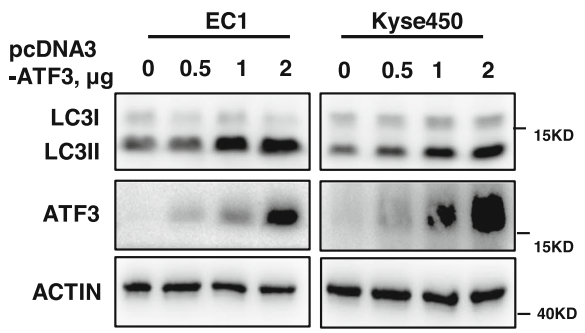

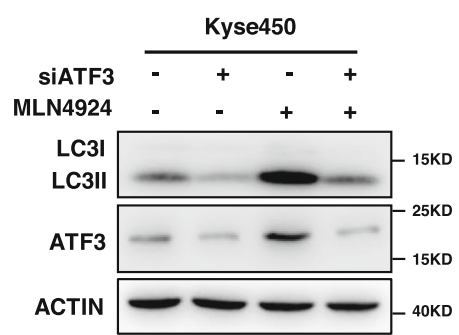

Fig. 2 ATF3 silencing blocked MLN4924-induced autophagy. a Treatment of MLN4924 induced the conversion of LC3-I to LC3-II in a dose dependent manner in both EC1 and Kyse450 cells. Cells were treated with the indicated concentrations of MLN4924 for $48 \mathrm{~h}$ and cells were collected and subjected to IB analysis for the expression of LC3. Actin was used as an equal loading control. b MLN4924 induced the conversion of LC3-I to LC3-II in a time-dependent manner in both EC1 and Kyse450 cells. Cells were treated with the $0.6 \mu$ mol/L of MLN4924 for indicated time and then cells were collected and subjected to IB analysis for the expression of LC3. Actin was used as an equal loading control. c-d Autophagic flux analysis. EC1 and Kyse450 cells, treated with DMSO or MLN4924 for 48 h, were incubated with or without CQ (50 $\mu \mathrm{M})$, BafA1 (50 $\mathrm{nM}$ ) or $3 \mathrm{MA}(5 \mathrm{mM})$ for $8 \mathrm{~h}$. The treated cells were then collected and subjected to IB analysis. Actin was used as an equal loading control. e-f ATF3 is required for MLN4924 induced autophagy in esophageal cancer cells. EC1 and Kyse450 cells were transfected with control or ATF3 siRNA for $48 \mathrm{~h}$ and then treated with $0.6 \mu \mathrm{mol} / \mathrm{L}$ MLN4924 for $48 \mathrm{~h}$. ATF3 Knockdown efficiency and conversion of LC3-I to LC3-II were assessed by IB analysis. g ATF3 overexpression increase autophagy. EC1 and Kyse450 cells were transfected with pcDNA3-ATF3 $(0 \mu \mathrm{g}, 0.5 \mu \mathrm{g}, 1 \mu \mathrm{g}, 2 \mu \mathrm{g})$ for $48 \mathrm{~h}$. ATF3 overexpression efficiency and conversion of LC3-I to LC3-II were assessed by IB analysis

in cancer cells $[35,36]$. Based on these findings, we determined whether MLN4924-induced ATF3 accumulation is mediated by ROS. We firstly detected cellular ROS level with the cell permeable ROS indicator, 2', 7'dichlorodihydrofuorescein diacetate (H2-DCFDA), and found that MLN4924 significantly induced ROS production in both EC1 and Kyse450 cells (Fig. 4a). Furthermore, we determined the role of ROS in MLN4924-induced ATF3 expression and autophagy. As shown in Fig. 4b and c, pre-treatment of esophageal cancer cells with NAC, a classical ROS scavenger, dramatically attenuated the expression of ATF3 (Fig. 4b) and inhibited the autophagic response (Fig. 4c) induced by MLN4924.

Given that Catalase and SOD1, as ubiquitous antioxidant enzymes, could inhibit the generation of ROS [37], we determine whether MLN4924 regulates the expression of Catalase or SOD1. As shown in Fig. 4d- 
A

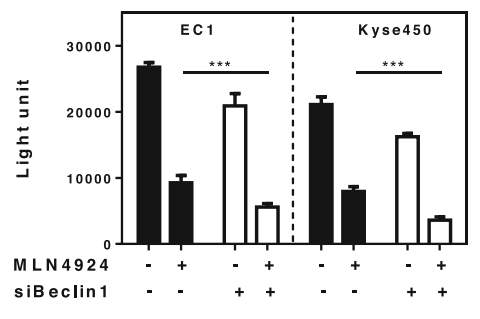

C

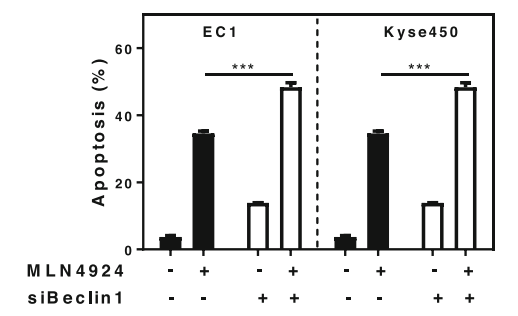

E

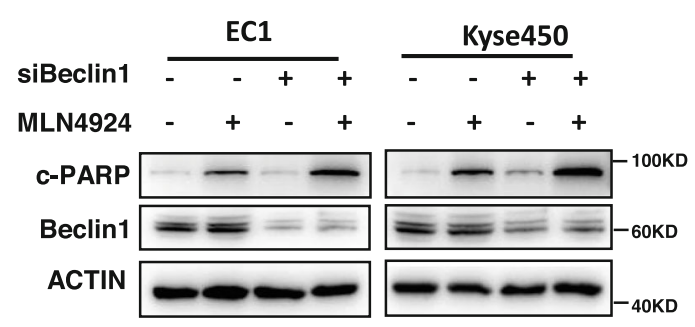

G

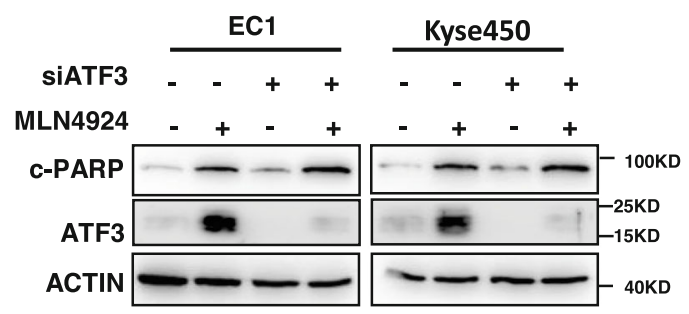

B

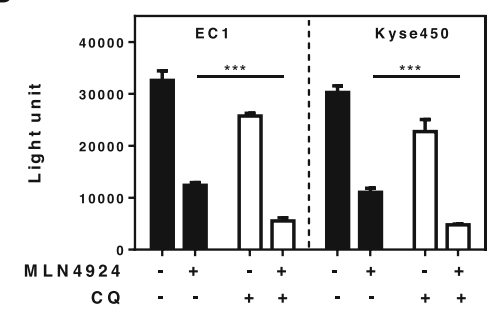

D

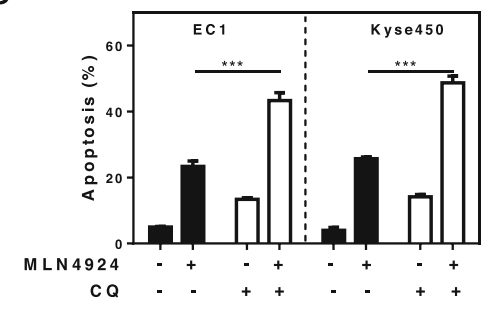

F

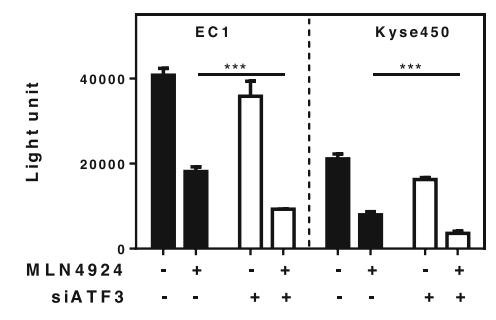

H

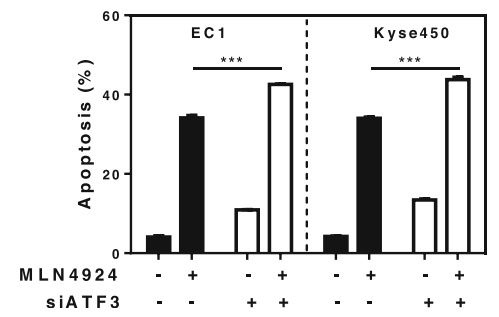

Fig. 3 MLN4924-induced autophagy was a survival signal and ATF3 silencing enhanced drug toxicity via inducing apoptosis. a-b The proliferation inhibition by MLN4924 was significantly increased by simultaneously blocking autophagy with siBeclin 1 (a) or CQ(b). The combination of siBeclin1 or CQ with MLN4924 in EC1 and Kyse450 cells significantly increased proliferation inhibition by ATPLite assay. c-d Apoptosis induced by MLN4924 was significantly increased by simultaneously blocking autophagy with siBeclin1 or CQ. The combination of siBeclin1(c)or CQ (d) with MLN4924 in EC1 and Kyse450 cells significantly increased apoptosis by Annexin V /PI double staining. e Beclin1 knockdown increased cleaved PARP expression induced by MLN4924. EC1 and Kyse450 cells were transfected with control or Beclin1 siRNA for $48 \mathrm{~h}$ and then treated with $0.6 \mu \mathrm{mol} / \mathrm{L}$ MLN4924 for $48 \mathrm{~h}$. Knockdown efficiency and cleaved PARP were assessed by IB analysis. $\mathbf{f}$-h Blocking autophagy by siATF3 remarkably suppressed cell proliferation and induced cell apoptosis compared with MLN4924 alone. EC1 and Kyse450 cells were transfected with control or ATF3 siRNA for $48 \mathrm{~h}$ and then treated with $0.6 \mu \mathrm{mol} / \mathrm{L}$ MLN4924 for $48 \mathrm{~h}$. Cell proliferation suppressed by MLN4924 was further significantly decreased by simultaneously silencing of ATF3 expression (f). Knockdown efficiency and cleaved PARP were assessed by IB analysis (g). Apoptosis detection by either annexin $\mathrm{V}$ and $\mathrm{PI}$ double staining $(\mathbf{h})$

e, MLN4924 significantly reduced the expression of Catalase at protein and mRNA levels but do not reduce the expression of SOD1. In order to determine the role of Catalase in MLN4924-induced ROS production and autophagy, Catalase was ectopically expressed in EC1 and Kyse450 cells with or without MLN4924 treatment. Indeed, Catalase expression significantly reversed MLN4924-induced ROS production (Fig. 4f). Moreover, Catalase expression completely blocked the conversion of LC3-I to LC3-II upon MLN4924 treatment (Fig. 4g). These findings demonstrated that MLN4924 induced ROS/ATF3 axis to trigger autophagy by reducing Catalase expression in esophageal cancer cells. 


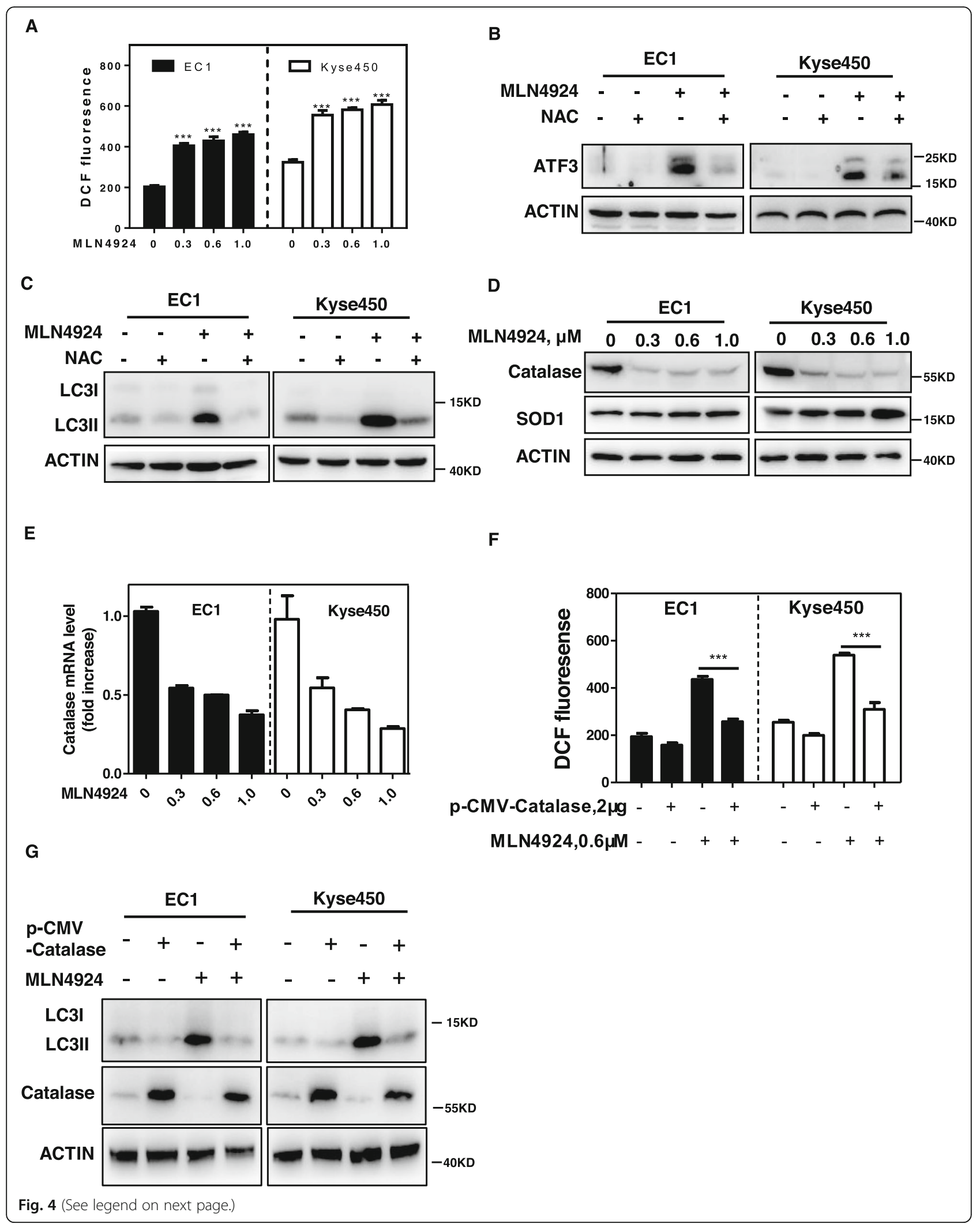


(See figure on previous page.)

Fig. 4 ATF3 accumulation is mediated by ROS upon MLN4924 treatment. a MLN4924 treatment induced ROS generation. EC1 and Kyse450 cells were treated with different dose of MLN4924 for $24 \mathrm{~h}$ and ROS generation was determined by H2-DCFDA staining. b NAC, a classical ROS scavenger, attenuated ATF3 expression. EC1 and Kyse450 cells were treated with $0.6 \mu \mathrm{mol} / \mathrm{L}$ MLN4924 alone or MLN4924 + NAC for $48 \mathrm{~h}$ and subjected to IB analysis for the expression of ATF3. Actin was used as an equal loading control. c Reduction of ROS by NAC significantly inhibited MLN4924-induced autophagy. EC1 and Kyse450 Cells were treated with $0.6 \mu \mathrm{mol} / \mathrm{L}$ of MLN4924 alone or MLN4924 + NAC for $48 \mathrm{~h}$ and subjected to IB analysis for the expression of LC3. Actin was used as an equal loading control. $\mathbf{d}$ MLN4924 decreased the expression of Catalase and did not affect the expression of SOD1. EC1 and Kyse450 Cells were treated with the indicated concentrations of MLN4924 for $48 \mathrm{~h}$ and subjected to IB analysis for the expression of Catalase, SOD1. Actin was used as an equal loading control. e MLN4924 decreased Catalase at the transcriptional level. EC1 and Kyse450 cells were treated with different dose of MLN4924 for $24 \mathrm{~h}$ and RNA were extracted and analyzed by qPCR (normalized to GAPDH). $\mathbf{f}$ Catalase overexpression attenuated MLN4924-induced ROS production. EC1 and Kyse450 cells were transfected with $2 \mu \mathrm{g}$ p-CMVCatalase for $24 \mathrm{~h}$ and then treated with $0.6 \mu \mathrm{mol} / \mathrm{L}$ MLN4924 for $24 \mathrm{~h}$. ROS generation was determined by H2-DCFDA staining. $\mathbf{g}$ Catalase overexpression reversed MLN4924-induced autophagy. EC1 and Kyse450 cells were transfected with 2 mg p-CMV-Catalase for $24 \mathrm{~h}$ and then treated with $0.6 \mu \mathrm{mol} / \mathrm{L}$ MLN4924 for $24 \mathrm{~h}$. Catalase overexpression efficiency and conversion of LC3-I to LC3-II were assessed by IB analysis

\section{MLN4924 activated autophagy by modulating NF-KB- catalase-ATF3 axis}

Since Catalase serves as a classical target gene of NF- $\mathrm{kB}$, we next determined whether the inhibitory effect of MLN4924 on Catalase expression and ATF3-induced autophagy is mediated by NF- $\mathrm{kB}$ pathway. Translocation of NF- $\mathrm{kB}$ to the nucleus is allowed by the phosphorylation of $I \kappa B \alpha$, resulting in its ubiquitination and degradation by CRLs complex [38]. As expected, MLN4924 treatment significantly inhibited global protein neddylation and Cullin1 neddylation, indicating the inactivation of CRL E3 ligase. Consequently, $\mathrm{p}-\mathrm{I}_{\kappa} \mathrm{B} \alpha$, a classical substrate of CRL, was dramatically accumulated upon neddylation-CRL inactivation (Fig. 5a). Moreover, IkB $\alpha$ knockdown markedly attenuated MLN4924-induced reduction of Catalase, accumulation of ATF3 and induction of autophagy (Fig. 5b). We further explored whether I $\mathrm{B} \alpha$ knockdown has the similar role of ATF3 knockdown-enhanced MLN4924-induced proliferation inhibition in EC1 and Kyse450 cells. As shown in Fig. 5c, IкB $\alpha$ knockdown also significantly increased MLN4924-induced proliferation inhibition. These findings collectively demonstrated that MLN4924 inhibited NF- $\kappa \mathrm{B}$ pathway to reduce Catalase expression, which promoted the ROS generation to eventually induce ATF3 accumulation and autophagic response.

\section{Discussion}

Esophageal cancer is the fourth largest malignant tumor of digestive system and sixth mortality rate of cancer diseases, while the novel therapeutic strategies for this deadly disease are urgently needed. Recently, protein neddylation pathway has emerged as a promising antiESCC target, as best supported by the discovery of overactivation of the neddylation pathway in esophageal cancer and the potent anti-ESCC effects of specific NAE inhibitor MLN4924 in preclinical trials. In the present study, we reported for the first time to our knowledge that MLN4924 treatment induced the accumulation of
ATF3 and ATF3-mediated autophagy via NF-кBCatalase-ROS pathway. Functionally, blockage of MLN4924-induced protective autophagy by ATF3 deletion sensitized ESCC cells to MLN4924-induced apoptosis. These preclinical findings indicated that NF$\mathrm{kB}$-Catalase-ROS-ATF3 axis served as a new protective autophagy mechanism in esophageal cancer upon MLN4924 treatment, which provided a rationale for combinational anti-ESCC therapy with dual inhibition of neddylation and autophagy pathways.

ATF3 is one of the crucial stress-responsive factors and acts as either a tumor suppressor or oncoprotein in cancer-type dependent manner [32, 39-42]. Generally, previous study has shown that ATF3 induced NOXA expression to promote apoptosis in chronic myelogenous leukemia (CML) cells [41]. Conversely, ATF3 could also promote proliferation of Adult T-cell leukemia (ATL) cells via mechanisms including upregulation of CDC2 and cyclin E2 [39]. Therefore, determining the role of ATF3 in ESCC development might provide potential intervention points for anti-ESCC therapy.

In this study, we found that MLN4924 treatment induced ATF3 expression to trigger autophagic response as a pro-survival signal while ATF3 silencing promoted cell apoptosis. Consistently, TCGA RNA-seq database analysis showed that ATF3 was substantially elevated in ESCC tissues when compared with the adjacent normal tissues (Fig. S1.A). Furthermore, high expression of ATF3 was negatively correlated with the prognostic survival curve in patients with esophageal cancer, suggesting that ATF3 may act as an oncogene in esophageal cancer (Fig. S1.B). Thus, our findings that MLN4924-induced ATF3 activation acts as a prosurvival event in ESCC highlights the therapeutic value of targeting ATF3 and neddylation pathway for combinational ESCC therapy.

ATF3 has been reported to regulate the expression of NF- $\kappa$ B target genes, such as TNF-a and IL-6 [43, 44]. Conversely, there are reports showing that NF-kB could 

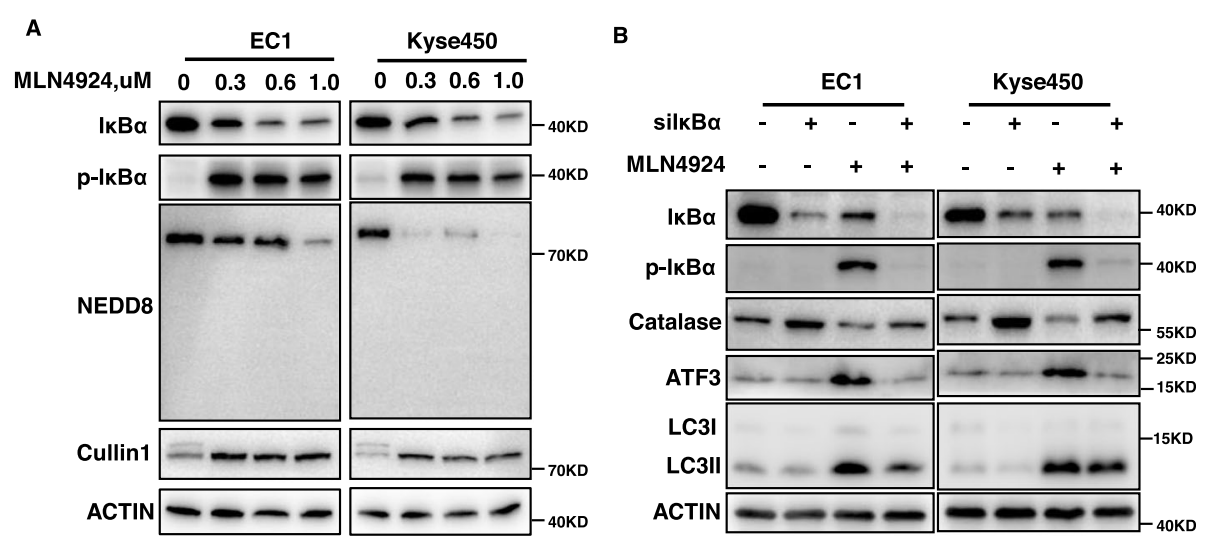

C

D
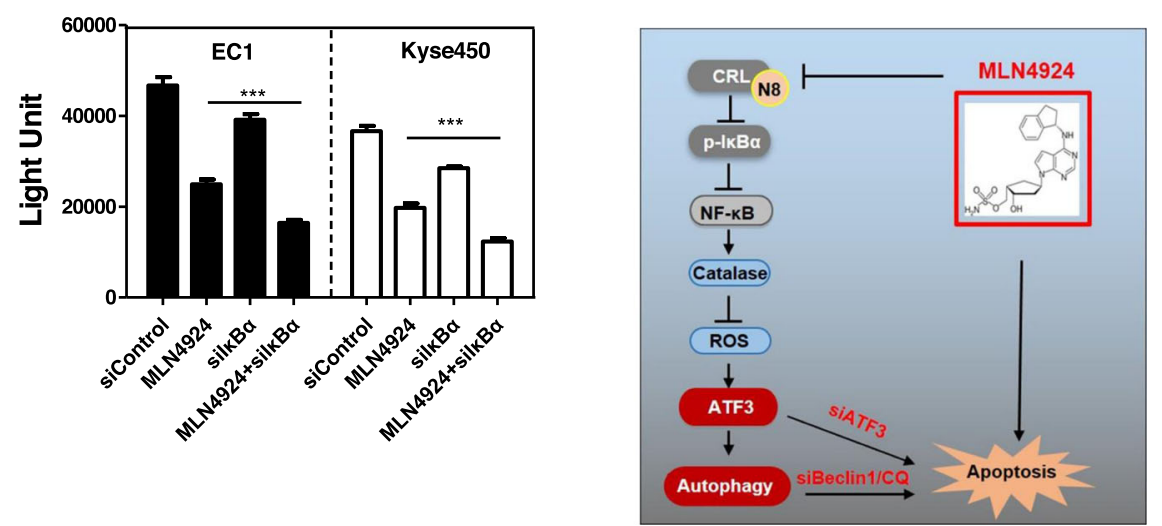

Fig. 5 MLN4924 activated autophagy by modulating NF-KB-Catalase-ATF3 axis. a MLN4924 inhibited the activation of NF-KB pathway. EC1 and Kyse450 Cells were treated with the indicated concentrations of MLN4924 for $48 \mathrm{~h}$ and subjected to IB analysis for the expression of IKBa, $\mathrm{p}$-IKBa, NEDD8. Actin was used as an equal loading control. b The effect of inhibition of NF-KB pathway caused by MLN4924 treatment was on both Catalase and ATF3 expression. EC1 and Kyse450 cells were transfected with control or silkBa for $24 \mathrm{~h}$ and then treated with $0.6 \mu \mathrm{mol} / \mathrm{L}$ MLN4924 for $48 \mathrm{~h}$. Knockdown efficiency and IKBa, p-IKBa and catalase were assessed by IB analysis. c Downregulation of IKBa by silkBa remarkably increased proliferation inhibition compared with MLN4924 alone. EC1 and Kyse450 cells were transfected with control or IkBa siRNA for $48 \mathrm{~h}$ and then treated with $0.6 \mathrm{\mu mol} / \mathrm{L}$ MLN4924 for $48 \mathrm{~h}$. $\mathbf{d}$ Schema of the mechanism for MLN4924-induced protective autophagy in ESCC

directly induce the transcriptional activation of ATF3 [45]. In the present study, however, we showed that ATF3 was activated by MLN4924-induced ROS due to the inhibition of NF- $\mathrm{kB}$-Catalase axis in ESCC cells, revealing a new mechanism by which NF- $\mathrm{kB}$ regulates ATF3. Further investigation of the cross-talk between ATF3 and NF- $\kappa B$ signaling is of great interest.

$\mathrm{NF}-\mathrm{kB}$ acts as a potent pro-survival transcription factor and promotes the progression of various tumors [46-48]. However, in the present study, we found that inactivation of NF- $k B$ pathway upon MLN4924 treatment acts as a protective role by inducing the ATF3-mediated autophagy in esophageal cancer cells. Consistently, ІкB $\alpha$ knockdown activates NF- $\kappa B$ signaling to attenuate MLN4924-induced ATF3 expression and protective autophagy. Together, NF- $k B$ pathway may have dual functions in response to therapeutic stresses, acting as a pro-death or a pro-survival signal upon treatment.

Previous studies have reported that ATF3 could induce autophagy. For example, LYN-1604, as a novel activator of ULK1, obviously up-regulated ATF3 to induce autophagy in triple negative breast cancer $[49,50]$. However, the further mechanism by which ATF3 induces autophagy is largely unknown. In the present study, we showed that MLN4924 induced autophagy dependent on ATF3 accumulation. These findings establish the necessity to explore the mechanism by which ATF3 promotes autophagy in future studies.

Our study suggested the following working model (Fig. 5d). In esophageal cancer cells, MLN4924 induces the expression of ATF3 by modulating NF- $\mathrm{kB}-$ Catalase-ROS 
pathway to trigger pro-survival autophagy, whereas targeting ATF3 blocks the autophagic response upon neddylation inhibition and thus sensitizes cancer cells to MLN4924-induced apoptosis. These findings provide a potential combination strategy of dually targeting ATF3 and neddylation pathway for effective anti-ESCC therapy.

\section{Supplementary information}

Supplementary information accompanies this paper at https://doi.org/10. 1186/s12964-020-00576-z.

Additional file 1. (PPTX $156 \mathrm{~kb}$ )

\section{Abbreviations}

NEDD8: neural precursor cell expressed developmentally down-regulated 8; CRL: Cullin-RING E3 ligase; ESCC: Esophageal squamous cell carcinoma; CHX: cycloheximide; CQ: chloroquine; BafA1: Bafilomycin A1; 3MA: 3methyladenine; IB: immunoblotting

\section{Acknowledgements}

We would like to thank Prof. Dakang Xu (Ruijin Hospital, Shanghai Jiaotong University School of Medicine, Shanghai) for providing us the pcDNA3-ATF3 plasmid.

\section{Authors' contributions}

LYP and $J\lrcorner$ conceived the general framework of this study and designed experiments. LYP, JYY, JX and CP performed experiments. LLH and HYQ provided technical or material support. LYP, JLJ, CLL and ZWJ prepared the manuscript. $\lrcorner\lrcorner$ supervised this study. All authors read and approved the final manuscript.

\section{Funding}

This work was supported by the following funds: The Chinese Minister of Science and Technology grant (2016YFA0501800), National Natural Science Foundation of China (Grants 81820108022, 81625018, 81802743), Program of Shanghai Academic/Technology Research Leader (18XD1403800), Innovation Program of Shanghai Municipal Education Commission (2019-01-07-00-10E00056) and National Thirteenth Five-Year Science and Technology Major Special Project for New Drug and Development (2017ZX09304001).

\section{Availability of data and materials}

The datasets used and/or analyzed during the current study are available from the corresponding author on reasonable request.

\section{Ethics approval and consent to participate}

Not applicable.

\section{Consent for publication}

We have obtained consent to publish this paper from the participants of this study.

\section{Competing interests}

The authors declare that they have no competing interests.

\section{Author details}

${ }^{1}$ Cancer Institute, Longhua Hospital, Shanghai University of Traditional Chinese Medicine, Shanghai 200032, China. ${ }^{2}$ School of Basic Medical Sciences, Zhengzhou University, Zhengzhou 450001, China. ${ }^{3}$ Department of Breast Surgery, Key Laboratory of Breast Cancer in Shanghai, Fudan University Shanghai Cancer Center, Shanghai, China.
Received: 12 December 2019 Accepted: 6 April 2020

Published online: 12 May 2020

\section{References}

1. Chen P, Hu T, Liang Y, Li P, Chen X, Zhang J, et al. Neddylation inhibition activates the extrinsic apoptosis pathway through ATF4-CHOP-DR5 Axis in human esophageal Cancer cells. Clin Cancer Res. 2016:22(16):4145-57.

2. Zhao Y, Morgan MA, Sun Y. Targeting Neddylation pathways to inactivate cullin-RING ligases for anticancer therapy. Antioxid Redox Signal. 2014 21(17):2383-400

3. Rabut G, Peter M. Function and regulation of protein neddylation. 'Protein modifications: beyond the usual suspects' review series. EMBO Rep. 2008; 9(10):969-76.

4. Li L, Wang M, Yu G, Chen P, Li H, Wei D, et al. Overactivated neddylation pathway as a therapeutic target in lung cancer. J Natl Cancer Inst. 2014; 106(6):dju083.

5. Soucy TA, Smith PG, Milhollen MA, Berger AJ, Gavin JM, Adhikari S, et al. An inhibitor of NEDD8-activating enzyme as a new approach to treat cancer. Nature. 2009;458(7239):732-6.

6. Xie P, Zhang M, He S, Lu K, Chen Y, Xing G, et al. The covalent modifier Nedd 8 is critical for the activation of Smurf1 ubiquitin ligase in tumorigenesis. Nat Commun. 2014;5:3733.

7. Gao Q, Yu GY, Shi JY, Li LH, Zhang WJ, Wang ZC, et al. Neddylation pathway is up-regulated in human intrahepatic cholangiocarcinoma and serves as a potential therapeutic target. Oncotarget. 2014;5(17):7820-32.

8. Xu J, Li L, Yu G, Ying W, Gao Q, Zhang W, et al. The neddylation-cullin 2RBX1 E3 ligase axis targets tumor suppressor RhoB for degradation in liver cancer. Mol Cell Proteomics. 2015:14(3):499-509.

9. Luo Z, Yu G, Lee HW, Li L, Wang L, Yang D, et al. The Nedd8-activating enzyme inhibitor MLN4924 induces autophagy and apoptosis to suppress liver cancer cell growth. Cancer Res. 2012;72(13):3360-71.

10. Yang D, Li L, Liu H, Wu L, Luo Z, Li H, et al. Induction of autophagy and senescence by knockdown of ROC1 E3 ubiquitin ligase to suppress the growth of liver cancer cells. Cell Death Differ. 2013;20(2):235-47.

11. Brownell JE, Sintchak MD, Gavin JM, Liao H, Bruzzese FJ, Bump NJ, et al. Substrate-assisted inhibition of ubiquitin-like protein-activating enzymes: the NEDD8 E1 inhibitor MLN4924 forms a NEDD8-AMP mimetic in situ. Mol Cell. 2010;37(1):102-11

12. Nawrocki ST, Griffin P, Kelly KR, Carew JS. MLN4924: a novel first-in-class inhibitor of NEDD8-activating enzyme for cancer therapy. Expert Opin Investig Drugs. 2012;21(10):1563-73.

13. Liao H, Liu XJ, Blank JL, Bouck DC, Bernard H, Garcia K, et al. Quantitative proteomic analysis of cellular protein modulation upon inhibition of the NEDD8-activating enzyme by MLN4924. Mol Cell Proteomics. 2011;10(11): M111009183.

14. Kim W, Bennett EJ, Huttlin EL, Guo A, Li J, Possemato A, et al. Systematic and quantitative assessment of the ubiquitin-modified proteome. Mol Cell. 2011:44(2):325-40

15. Wang $Y$, Luo Z, Pan $Y$, Wang $W$, Zhou $X$, Jeong $L S$, et al. Targeting protein neddylation with an NEDD8-activating enzyme inhibitor MLN4924 induced apoptosis or senescence in human lymphoma cells. Cancer Biol Ther. 2015; 16(3):420-9.

16. Blank JL, Liu XJ, Cosmopoulos K, Bouck DC, Garcia K, Bernard H, et al. Novel DNA damage checkpoints mediating cell death induced by the NEDD8activating enzyme inhibitor MLN4924. Cancer Res. 2013;73(1):225-34.

17. Godbersen JC, Humphries LA, Danilova OV, Kebbekus PE, Brown JR, Eastman A, et al. The Nedd8-activating enzyme inhibitor MLN4924 thwarts microenvironment-driven NF-kappaB activation and induces apoptosis in chronic lymphocytic leukemia B cells. Clin Cancer Res. 2014;20(6):1576-89.

18. Lin JJ, Milhollen MA, Smith PG, Narayanan U, Dutta A. NEDD8-targeting drug MLN4924 elicits DNA rereplication by stabilizing Cdt1 in S phase, triggering checkpoint activation, apoptosis, and senescence in cancer cells. Cancer Res. 2010:70(24):10310-20.

19. Milhollen MA, Narayanan U, Soucy TA, Veiby PO, Smith PG, Amidon B. Inhibition of NEDD8-activating enzyme induces rereplication and apoptosis in human tumor cells consistent with deregulating CDT1 turnover. Cancer Res. 2011:71(8):3042-51.

20. Soucy TA, Dick LR, Smith PG, Milhollen MA, Brownell JE. The NEDD8 conjugation pathway and its relevance in Cancer biology and therapy. Genes Cancer. 2010;1(7):708-16. 
21. Milhollen MA, Traore T, Adams-Duffy J, Thomas MP, Berger AJ, Dang L, et al. MLN4924, a NEDD8-activating enzyme inhibitor, is active in diffuse large Bcell lymphoma models: rationale for treatment of NF-\{kappa\}B-dependent lymphoma. Blood. 2010;116(9):1515-23.

22. Jiang $Y$, Liang $Y$, Li L, Zhou L, Cheng $W$, Yang $X$, et al. Targeting neddylation inhibits intravascular survival and extravasation of cancer cells to prevent lung-cancer metastasis. Cell Biol Toxicol. 2019;35(3):233-45.

23. Zhang W, Liang Y, Li L, Wang X, Yan Z, Dong C, et al. The Nedd8-activating enzyme inhibitor MLN4924 (TAK-924/Pevonedistat) induces apoptosis via cMyc-Noxa axis in head and neck squamous cell carcinoma. Cell Prolif. 2019; 52(2):e12536.

24. Zhou L, Jiang Y, Luo Q, Li L, Jia L. Neddylation: a novel modulator of the tumor microenvironment. Mol Cancer. 2019;18(1):77.

25. Luo Z, Pan Y, Jeong LS, Liu J, Jia L. Inactivation of the Cullin (CUL)-RING E3 ligase by the NEDD8-activating enzyme inhibitor MLN4924 triggers protective autophagy in cancer cells. Autophagy. 2012;8(11):1677-9.

26. Zhao Y, Xiong X, Jia L, Sun Y. Targeting Cullin-RING ligases by MLN4924 induces autophagy via modulating the HIF1-REDD1-TSC1-mTORC1-DEPTOR axis. Cell Death Dis. 2012;3:e386.

27. Zhao Y, Sun Y. Targeting the mTOR-DEPTOR pathway by CRL E3 ubiquitin ligases: therapeutic application. Neoplasia. 2012;14(5):360-7.

28. Yang D, Zhao Y, Liu J, Sun Y, Jia L. Protective autophagy induced by RBX1/ ROC1 knockdown or CRL inactivation via modulating the DEPTOR-MTOR axis. Autophagy. 2012;8(12):1856-8.

29. Hai T, Hartman MG. The molecular biology and nomenclature of the activating transcription factor/cAMP responsive element binding family of transcription factors: activating transcription factor proteins and homeostasis. Gene. 2001;273(1):1-11.

30. You Z, Xu J, Li B, Ye H, Chen L, Liu Y, et al. The mechanism of ATF3 repression of epithelial-mesenchymal transition and suppression of cell viability in cholangiocarcinoma via p53 signal pathway. J Cell Mol Med. 2019;23(3):2184-93.

31. Yin X, Dewille JW, Hai T. A potential dichotomous role of ATF3, an adaptiveresponse gene, in cancer development. Oncogene. 2008;27(15):2118-27.

32. Perez $\mathrm{S}$, Vial E, van Dam H, Castellazzi M. Transcription factor ATF3 partially transforms chick embryo fibroblasts by promoting growth factorindependent proliferation. Oncogene. 2001;20(9):1135-41.

33. Yuan X, Yu L, Li J, Xie G, Rong T, Zhang L, et al. ATF3 suppresses metastasis of bladder cancer by regulating gelsolin-mediated remodeling of the actin cytoskeleton. Cancer Res. 2013:73(12):3625-37.

34. Mo P, Wang H, Lu H, Boyd DD, Yan C. MDM2 mediates ubiquitination and degradation of activating transcription factor 3. J Biol Chem. 2010;285(35): 26908-15.

35. Hoetzenecker W, Echtenacher B, Guenova E, Hoetzenecker K, Woelbing F, Bruck J, et al. ROS-induced ATF3 causes susceptibility to secondary infections during sepsis-associated immunosuppression. Nat Med. 2011;18(1):128-34.

36. Chen P, Hu T, Liang Y, Jiang Y, Pan Y, Li C, et al. Synergistic inhibition of autophagy and neddylation pathways as a novel therapeutic approach for targeting liver cancer. Oncotarget. 2015;6(11):9002-17.

37. Nishikawa M, Hashida M, Takakura Y. Catalase delivery for inhibiting ROSmediated tissue injury and tumor metastasis. Adv Drug Deliv Rev. 2009; 61(4):319-26.

38. Tan P, Fuchs SY, Chen A, Wu K, Gomez C, Ronai Z, et al. Recruitment of a ROC1-CUL1 ubiquitin ligase by Skp1 and HOS to catalyze the ubiquitination of I kappa B alpha. Mol Cell. 1999:3(4):527-33.

39. Hagiya K, Yasunaga J, Satou Y, Ohshima K, Matsuoka M. ATF3, an HTLV-1 bZip factor binding protein, promotes proliferation of adult T-cell leukemia cells. Retrovirology. 2011;8:19.

40. Udayakumar TS, Stoyanova R, Shareef MM, Mu Z, Philip S, Burnstein KL, et al. Edelfosine promotes apoptosis in androgen-deprived prostate tumors by increasing ATF3 and inhibiting androgen receptor activity. Mol Cancer Ther. 2016;15(6):1353-63.

41. Kuroda J, Yamamoto M, Nagoshi H, Kobayashi T, Sasaki N, Shimura Y, et al. Targeting activating transcription factor 3 by Galectin- 9 induces apoptosis and overcomes various types of treatment resistance in chronic myelogenous leukemia. Mol Cancer Res. 2010:8(7):994-1001.

42. Zhou J, Edgar BA, Boutros M. ATF3 acts as a rheostat to control JNK signalling during intestinal regeneration. Nat Commun. 2017;8:14289.

43. Wu YP, Cao C, Wu YF, Li M, Lai TW, Zhu C, et al. Activating transcription factor 3 represses cigarette smoke-induced IL6 and IL8 expression via suppressing NF-kappaB activation. Toxicol Lett. 2017;270:17-24.
44. Park SH, Kim J, Do KH, Park J, Oh CG, Choi HJ, et al. Activating transcription factor 3-mediated chemo-intervention with cancer chemokines in a noncanonical pathway under endoplasmic reticulum stress. J Biol Chem. 2014;289(39):27118-33.

45. Jack GD, Cabrera MC, Manning ML, Slaughter SM, Potts M, Helm RF. Activated stress response pathways within multicellular aggregates utilize an autocrine component. Cell Signal. 2007;19(4):772-81.

46. Tanaka H, Matsumura I, Ezoe S, Satoh Y, Sakamaki T, Albanese C, et al. E2F1 and c-Myc potentiate apoptosis through inhibition of NF-kappaB activity that facilitates MnSOD-mediated ROS elimination. Mol Cell. 2002;9(5):1017-29.

47. Pham CG, Bubici C, Zazzeroni F, Papa S, Jones J, Alvarez K, et al. Ferritin heavy chain upregulation by NF-kappaB inhibits TNFalpha-induced apoptosis by suppressing reactive oxygen species. Cell. 2004;119(4):529-42.

48. Duran A, Linares JF, Galvez AS, Wikenheiser K, Flores JM, Diaz-Meco MT, et al. The signaling adaptor p62 is an important NF-kappaB mediator in tumorigenesis. Cancer Cell. 2008;13(4):343-54.

49. Ouyang L, Zhang L, Fu L, Liu B. A small-molecule activator induces ULK1modulating autophagy-associated cell death in triple negative breast cancer. Autophagy. 2017;13(4):777-8.

50. Zhang L, Fu L, Zhang S, Zhang J, Zhao Y, Zheng Y, et al. Discovery of a small molecule targeting ULK1-modulated cell death of triple negative breast cancer in vitro and in vivo. Chem Sci. 2017:8(4):2687-701.

\section{Publisher's Note}

Springer Nature remains neutral with regard to jurisdictional claims in published maps and institutional affiliations.
Ready to submit your research? Choose BMC and benefit from:

- fast, convenient online submission

- thorough peer review by experienced researchers in your field

- rapid publication on acceptance

- support for research data, including large and complex data types

- gold Open Access which fosters wider collaboration and increased citations

- maximum visibility for your research: over $100 \mathrm{M}$ website views per year

At $\mathrm{BMC}$, research is always in progress.

Learn more biomedcentral.com/submissions 